\title{
Measurement of Plasma Etch Damage by a New Slow Trap Profiling Technique
}

\author{
Philip Tanner, Sima Dimitrijev, Member, IEEE, Yew-Tong Yeow, Senior Member, IEEE, H. Barry Harrison
}

\begin{abstract}
MOS capacitor structures with plasma damaged oxides have been used to demonstrate a new technique for profiling slow traps at the $\mathrm{Si}_{-} \mathrm{SiO}_{2}$ interface. The technique measures the density and trapping rate of slow traps by stepping the gate voltage in small increments and monitoring the resulting substrate current transients, thereby producing a profile of the traps in energy and response time. The response time is a function of the trap's energy position and distance from the interface. Some traps created by plasma etching are not obvious in quasistatic $C V$ measurements, yet are clearly evident when the new technique is used. Results show an increase in slow trap densities and response times in the upper half of the silicon bandgap with long plasma overetch times. In comparison, wet etched control devices show only low densities of slow traps with shorter response times around the midgap.
\end{abstract}

\section{INTRODUCTION}

$\mathbf{S}$ LOW interface traps in metal-oxide-semiconductor devices are important as they are thought to be the main source of $1 / f$ noise, random telegraph signals, and threshold voltage shifts in current and future MOSFETS [1]-[4]. These slow trapping centers are generally considered to be localized within the strained interfacial region up to $30 \AA$ from the Si$\mathrm{SiO}_{2}$ interface and exchange charge with the silicon at a rate which depends on the separation distance [4], [5]. Schwalke and Kerber reported long relaxation times of MOS capacitors after exposure to X-rays, suggesting that the primary cause was electron tunneling between the conduction band and slow traps in an energy state above the midgap [6].

Plasma etching is one of the processing steps thought to be responsible for the creation of both fast and slow interface traps as a result of charge build-up on isolated gate materials during overetching and ionizing radiation emmitted by the plasma [7], [8]. The spatial density and energy location of interface traps are often determined by well established capacitance-voltage measurement techniques, but these do not provide time resolution and, depending on the ramp rate used in the case of quasistatic $C V$ measurements, may not even detect the slower traps present in the oxide.

In this paper, we report on a slow interface trap profiling technique developed in our laboratory [9], [10] to monitor the density, energy location and response time of slow interface traps which are a direct result of the plasma etch process.

Manuscript received March 7, 1996; revised July 8, 1996. This work was supported by an Australian Research Council Collaborative grant and an equipment donation from LAM Research, Fremont, CA.

The authors are with the School of Microelectronic Engineering, Griffith University, Nathan, Queensland, Australia.

Publisher Item Identifier S 0741-3106(96)08310-3.
Briefly, the technique involves stepping the gate voltage of a MOS capacitor in small increments and recording the resulting substrate current transients. Fresh control samples provide transients lasting no longer than $30 \mathrm{~ms}$ at any position in the silicon bandgap, whereas devices which have been subjected to stress by constant current stressing or plasma etching exhibit considerably longer response times at certain energy positions. The value of the current at a time greater than $30 \mathrm{~ms}$ after a voltage step is a direct measure of the density of slow traps capturing or emitting charge at that instant. Slow traps can be resolved in energy and response time (related to distance from the interface), providing important additional information to compliment conventional techniques used to relate oxide damage to process conditions and interface quality.

\section{EXPERIMENTAL CONDITIONS}

The devices used in these experiments were of the structure polysilicon gate p-substrate MOS capacitors and were fabricated by standard MOS processes. The gate oxide is $400 \AA$-thick grown in a wet ambient at $800^{\circ} \mathrm{C}$ for $60 \mathrm{~min}$. Polysilicon plasma etching was conducted in a LAM490B parallel plate $\mathrm{RF}$ etcher using a $\mathrm{He} / \mathrm{SF}_{6}$ gas mix and an $\mathrm{RF}$ power of $100 \mathrm{~W}$ resulting in an etch time of $3 \mathrm{~min}$. Aluminum dots of area $0.015 \mathrm{~cm}^{2}$ were used as a mask to ensure uniform collection of charge from the plasma and endpoint detection with an accuracy of less than $1 \mathrm{~s}$ was achieved by means of an optical reflectance method from a point on the wafer within a few centimeters of the devices. Capacitors with plasma overetch times of 2, 12, and $30 \mathrm{~s}$ were produced and control devices of the same area were fabricated using standard wet etch techniques.

Quasistatic $C V$ curves were measured from strong inversion to accumulation at $40 \mathrm{mV} / \mathrm{s}$. Slow trap profiles were measured using an HP4145B parameter analyzer to step the gate voltage in $50 \mathrm{mV}$ steps and the substrate current transients after each gate voltage step were measured and digitized. The three measured parameters gate voltage, substrate current, and time since the voltage step (response time) form the basis for the slow trap profile. From this data, the slow trap distribution, $D_{\mathrm{st}}$, in $\mathrm{eV}^{-1} \cdot \mathrm{s}^{-1} \cdot \mathrm{cm}^{-2}$ at each point in the bandgap and instant in time is obtained by the equation $D_{\mathrm{st}}=I_{\mathrm{sub}} /(q . \Delta E . A)$ where $q$ is the electronic charge, $\Delta E$ is the change in surface Fermi level produced by the voltage step, and $A$ is the device area. At each gate voltage, the band bending and $\Delta E$ are derived from the quasistatic $C V$ curve. 


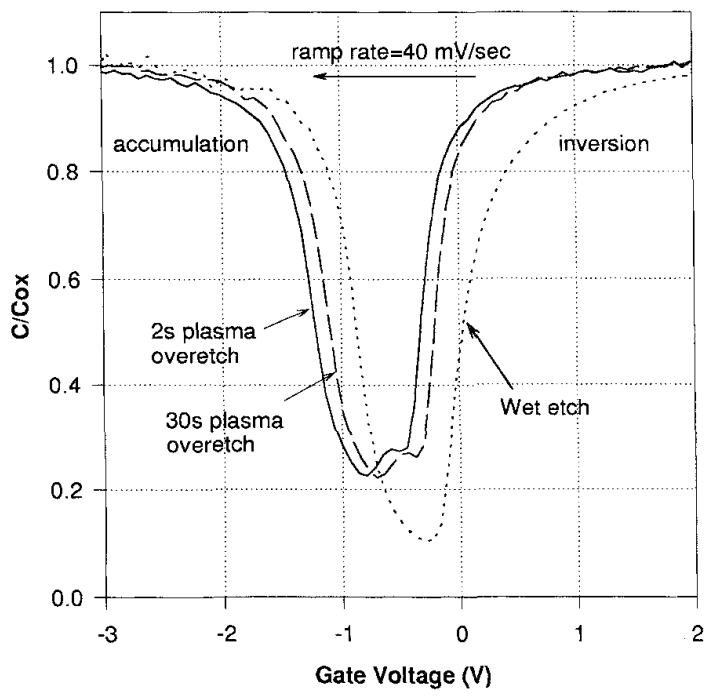

Fig. 1. Quasistatic curves of MOS capacitors manufactured by wet etching (-..), and plasma etching with $2 \mathrm{~s}$ (_ $)$ and $30 \mathrm{~s}(--)$ overetch.

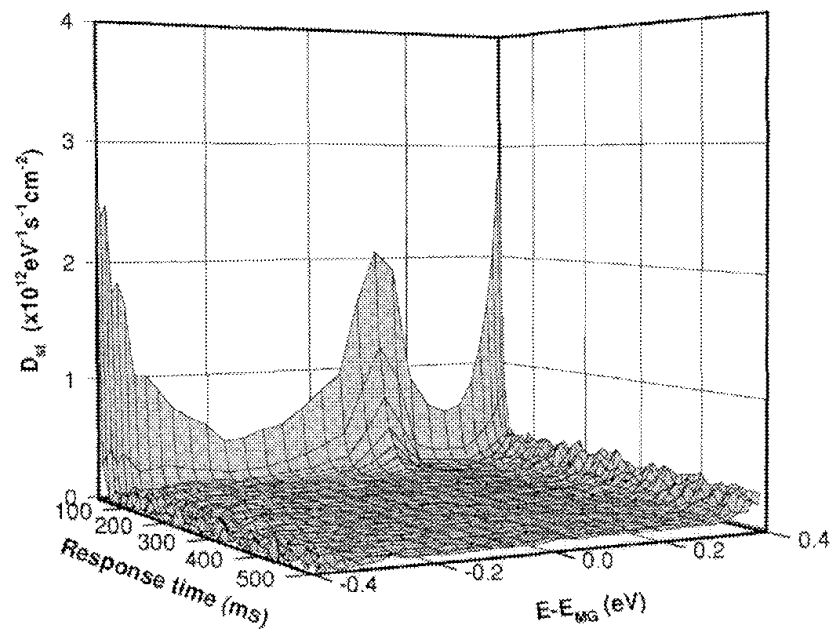

Fig. 2. Slow trap profile of plasma etched MOS capacitor after $2 \mathrm{~s}$ overetch time. The gate voltage was stepped in $50 \mathrm{mV}$ steps from accumulation to inversion. The energy axis is referred to the silicon midgap and the time axis begins at $30 \mathrm{~ms}$.

\section{RESULTS AND DISCUSSION}

The quasistatic $C V$ curves for the cases with $2 \mathrm{~s}$ and $30 \mathrm{~s}$ plasma overetch are shown in Fig. 1 along with that of a wet etched device. When compared with the wet etched curve, the plasma etched curves are shifted in the negative gate voltage direction indicating the presence of positive charge in the oxide. The distortion in the plasma etched curves indicates similar densities of interface traps for both cases, yet the slow trap profiles described below show that they are very different.

The slow trap profiles for these two devices are shown in Figs. 2 and 3 . The density of slow traps, $D_{\text {st }}$, is plotted as a function of energy position from midgap $\left(E-E_{\mathrm{MG}}\right)$ and the response time from 30 to $500 \mathrm{~ms}$. Due to the initial switching transient, the substrate current due to trapping can only be measured accurately after $30 \mathrm{~ms}$ from the gate voltage step. As others have found [6], no slow transients were detected in the

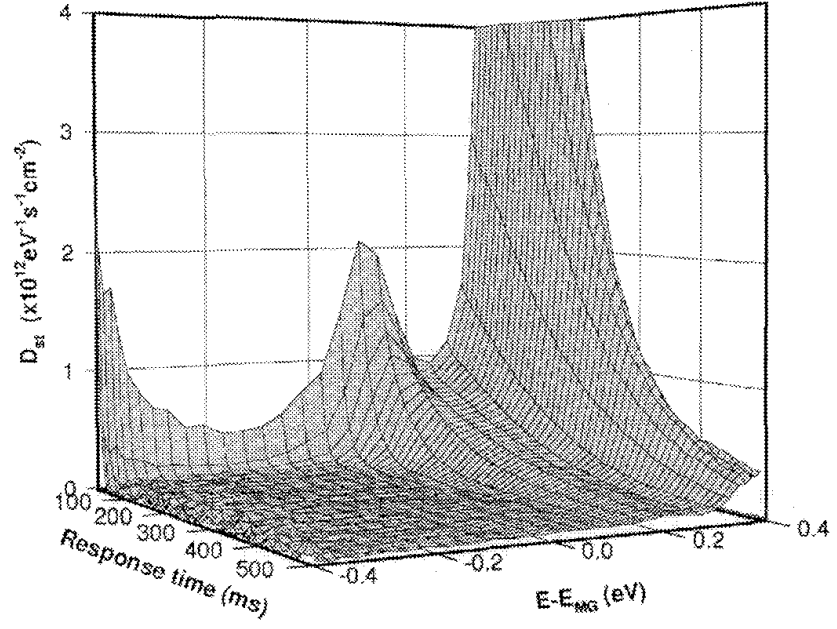

Fig. 3. Slow trap profile of plasma etched MOS capacitor after 30 s overetch time. The gate voltage was stepped in $50 \mathrm{mV}$ steps from accumulation to inversion. The energy axis is referred to the silicon midgap and the time axis begins at $30 \mathrm{~ms}$.

accumulation regions of each device. The corresponding plot for the wet etched sample (not shown) contained densitites of slow traps less than $3 \times 10^{11} \mathrm{eV}^{-1} \cdot \mathrm{s}^{-1} \cdot \mathrm{cm}^{-2}$ near the midgap, with maximum response times of approximately $100 \mathrm{~ms}$. The 2 s plasma overetched device (Fig. 2) contains an increased number of slow traps throughout the bandgap with a peak centered around $E_{\mathrm{MG}}+0.1 \mathrm{eV}$ with a maximum response time of $100 \mathrm{~ms}$. This peak coincides with the distortion mentioned earlier in the quasistatic $C V$ curves of Fig. 1. The $30 \mathrm{~s}$ profile (Fig. 3) shows the same peak at $E_{\mathrm{MG}}+0.1 \mathrm{eV}$ but has a longer response time. There is also a large increase in slower traps at energies closer to the silicon conduction band $\left(E_{\mathrm{MG}}+0.2 \mathrm{eV}\right.$ to $0.4 \mathrm{eV}$ ), with response times as long as $500 \mathrm{~ms}$. The quasistatic $C V$ plots (Fig. 1) show the two devices to have the same density of fast interface states. The slow trap profiles in Figs. 2 and 3 show that the longer overetch has induced more traps which also have much longer response times. The profile of the $12 \mathrm{~s}$ overetch case (not shown) revealed trap densities and response times between those of the $2 \mathrm{~s}$ and $30 \mathrm{~s}$ cases. It is very difficult to extract any contributions from the slower traps in the quasistatic $C V$ measurement as this part of the curve rises steeply to the oxide capacitance level as the inversion layer forms. Also, the ramp rate of $40 \mathrm{mV} / \mathrm{s}$ may be too fast for these slower traps to respond. It is interesting to note that the peak in the slow trap profile centered around $E_{\mathrm{MG}}+0.1 \mathrm{eV}$ closely resembles the peak created in a wet etched device after constant current stressing. It appears that part of the damage to the oxide during overetching can be said to be analogous to constant current stressing as deduced by others [6], [7] while another mechanism is responsible for the creation of slow traps at energies closer to the conduction band and more remote from the interface. The usefulness of the new technique was highlighted by similar experiments with thin gate oxides $(8 \mathrm{~nm})$ which revealed slow traps only in this high-energy region with none detected between midgap and $E_{\mathrm{MG}}+0.2 \mathrm{eV}$. No damage was evident in the quasistatic $C V$ curves. 


\section{CONCLUSIONS}

The new slow trap profiling technique has proven to be a useful tool in characterizing the changes in electronic and structural properties of the $\mathrm{Si}_{-} \mathrm{SiO}_{2}$ interface resulting from plasma etching by providing the density, energy, and response time of slow traps which were not clearly evident from the quasistatic $C V$ measurement. When compared to a wet etch control device, a very brief plasma overetch of $2 \mathrm{~s}$ produced an increase in slow trap densities at energies around $E_{\mathrm{MG}}+0.1 \mathrm{eV}$ with response times of up to $100 \mathrm{~ms}$, while progressively longer overetch times increased the response times of these traps and created traps at energies between 0.2 and $0.4 \mathrm{eV}$ above midgap which appear to extent further from the interface.

The oxide growth and processing conditions used here were not chosen to minimize trap creation. It is expected that highquality gate oxides and post etch annealing would reduce the damage considerably and further investigations are currently being undertaken.

\section{ACKNOWLEDGMENT}

The authors would like to acknowledge AWA Microelectronics, Sydney, Australia, for providing samples, and LAM Research, Fremont, CA, for providing the 490B plasma etcher.

\section{REFERENCES}

[1] A. Van der Ziel, "Flicker noise in electronic devices," Adv. Electron. Electron Phys., vol. 49, p. 225, 1979.

[2] K. K. Hung, P. K. Ko, C. Hu, and Y. C. Cheng, "Random telegraph noise of deep-submicron MOSFET's," IEEE Electron Device Lett., vol. 11, no. 2, p. 90,1990

[3] D. M. Fleetwood, P. S. Winokur, R. A. Reber, Jr., T. L. Meisenheimer, J. R. Schwank, M. R. Shaneyfelt, and L. C. Riewe, "Effects of oxide traps, interface traps, and border traps on metal-oxide-semiconductor devices," J. Appl. Phys., vol. 73, no. 10, p. 5058, 1993.

[4] T. L. Tewksbury III and H-S Lee, "Characterization, modeling, and minimization of transient threshold voltage shifts in MOSFET"s,"IEEE J. Solid-State Circuits, vol. 29, no. 3, p. 239, 1994.

[5] R. E. Paulsen, R. R. Siergiej, M. L. French, and M. H. White, "Observation of near-interface oxide traps with the charge-pumping technique," IEEE Electron Device Lett., vol. 13, no. 12, p. 627, 1992

[6] U. Schwalke and M. Kerber, "Effect of oxidation processing on the energy distribution and charging time of radiation induced interface traps," Appl. Phys. Lett., vol. 58, no. 16, p. 1774, 1991.

[7] H. Shin, N. Jha, X-Y. Qian, G. W. Hills, and C. Hu, "Plasma etching charge-up damage to thin oxides," Solid-State Technol., p. 29, Aug. 1993.

[8] S. Fang and J. P. McVittie, "Oxide damage from plasma charging: breakdown mechanism and oxide quality," IEEE Trans. Electron Devices, vol. 41, no. 6, p. $1034,1994$.

[9] P. Tanner, S. Dimitrijev, and H. B. Harrison, "Technique for monitoring slow interface trap characteristics in MOS capacitors," Electron. Lett. vol. 31 , no. 21 , p. $1880,1995$.

[10] S. Dimitrijev, P. Tanner, Z-Q. Yao, and H. B. Harrison, "Slow trap characterization by measurements of current-voltage characteristics of MOS capacitors," unpublished. 